(Aus der deutschen psychiatrischen Universitätsklinik in Prag [Prof. Dr. A. Pick].)

\title{
Die forensische Bedeutung der Amnesie und ihre Erklärung bei paranoiden Erkrankungen.
}

Von

Dr. Bruno Fiseher,

Assistent der Klinik.

Kriminelle Handlungen verschiedenster Art, wie Attentate auf einen vermeintlichen Gegner, Mord, Brandstiftung, Selbstverstümmelung gehören bei den paranoiden Erkrankungen nicht zu den Seltenheiten und finden ihre Erklärung in den entsprechenden geistigen Störungen. Nicht selten kommt es nach derartigen kriminellen Handlungen zu als Amnesie imponierenden Erscheinungen, die zwar in der Literatur wenig erwähnt, besonders aus forensischen Gründen doch gelegentlich von Bedeutung sein können.

Wassmund ${ }^{1}$ ) hat 1885 auf das Vorhandensein der Amnesie bei Paranoia in fünf Fällen allerdings mit wenig Erfolg hinzuweisen gesucht; in drei seiner Fälle handelt es sich gar nicht um Paranoia, während in den übrigen zwei Fällen die Amnesie einmal auf einem Trauma beruht, einmal ein auf einem nach einer schweren Blutung entstandenen Schwächezustande basierendes zufälliges Vorkommnis bildet, das ebenso gut auch beim normalen Menschen eintreten kann.

Schäffer (1893) erwähnt vielleicht als erster als eine bei Querulanten gewöhnliche Erscheinung, daß Kranke dieser Art Handlungen, welche sie selbst begangen haben, auf das Bestimmteste bestreiten. Wernicke führt Beispiele dafür bei Querulanten und verwandten $\mathrm{Zu}$ ständen chronischer partieller Psychosen an und auch Hitzig kennt diese Erscheinung, die er als charakteristisch für das Krankheitsbild der verrückten Querulanten ansieht. A. Pick macht gleichfalls in seiner Arbeit ${ }^{2}$ ) zur Psychologie des Vergessens bei Geistes- und Nervenkrankheiten auf Fälle dieser Psychosen aufmerksam, die fast alle mindestens Andeutungen eines Mangels an Reproduktionstreue aufweisen.

In der gerichtlichen Psychiatrie wurde dieser Erscheinung bei paranoischen Zuständen wohl mangels an einschlägigen Fällen nicht

1) Wassmund, Beitrag zur Frage von dem Vorkommen der kompletten und partiellen Amnesie bei der akuten und chronischen Paranoia. Inaug. Diss. Berlin 1886.

2) Arch. f. krim. Anthropologie.

Z. f. d. ges. gerichtl. Medizin. Bd. 1. 
die genügende Aufmerksamkeit geschenkt, obgleich gerade bei diesen die Frage der Vorspiegelung einer vorhandenen Amnesie stark in den Vordergrund tritt. Zeigt doch zumal der Paranoiker oft im ganzen Auftreten, Benehmen, namentlich im Beginn und in den leichteren Fällen keine auffallenden Störungen; er ist vollkommen korrekt, geordnet, hat sich sehr in der Gewalt, läßt von seinen durch die Wahnideen bedingten Affektstörungen nichts merken und wird vom Laien als verschlossener, verdrießlicher Mensch, als Sonderling beschrieben. Bei diesen' Kranken bilden die Wahnvorstellungen, wenn nicht das einzige, so doch das bei weitem hervorstechendste Krankheitssymptom, während ebenso die Klarheit wie die Ordnung im Denken, Wollen und Handeln vollkommen erhalten ist (Kraepelin). Sind schon gemeingefährliche Handlungen dieser dem Laien häufig ein Rätsel, so wird die Amnesie hierfür ihm überhaupt unverständlich, sie wird als unglaublich, als simuliert angesehen.

Das seltene Vorkommen derartiger einschlägiger Fälle, die Verschiedenfältigkeit der als Amnesie sich darstellenden Erscheinungen veranlaßt mich, im Folgenden vier Krankengeschichten von Fällen wiederzugeben, die, kriminell geworden, nach erfolgter Begutachtung durch Gerichtsärzte der Klinik zugingen.

I. J. T., 56 jähriger Mann, hereditär belastet; die Eltern werden als eigentümlich, bald sehr freundlich, bald streitsüchtig geschildert; 2 Geschwister geisteskrank, von denen ein Bruder lange Zeit wegen Schwachsinns in einer Anstalt interniert war, eine Schwester sehr streitsüchtig, die aus einem ganz unbedeutenden Grunde sich in selbstmörderischer Absicht ins Wasser stürzte. Er entwickelte sich in der Jugend ganz normal, war bis zu seiner Verheiratung im 45. Lebensjahre psychisch nicht auffallig, ein ruhiger, verläßlicher Arbeiter, stets versehlossen und wenig gesprächig. - Bald nach der Heirat (1902) kam es zu ehelichen Zwistigkeiten, er glaubte sich durch die Frau um seinen guten Ruf, seine Stellung gebracht; die ständige Kontrolle, die Behandlung seiner Kinder, denen sie den Verkehr mit ihm verbot und die sie, um ihn zu ärgern, oft vom Hause fernhielt, regten ihn sehr auf.

Durch Aufführung eines Hausbaues 1908 geriet er in finanzielle Schwierigkeiten, wurde von seiner Frau, die Vermögen besaß, im Stiche gelassen, mußte ihr sogar, als er die Scheidungsklage einreichte und damit abgewiesen wurde, 10 Kronen monatlich bezahlen. Seine Stimmung war dadurch gedrückt; er, der früber eine kaltblütige Ruhe bewahrte, wurde jetzt ein nervöser, scheuer, aufgeregter und reizbarer Mensch. Da er seinen monatlichen Zahlungsverpflichtungen nicht nachkommen konnte, ließ seine Frau auf dem Hause 70 Kronen für Sustentationskosten sicher. stellen und das erweckte in ihm den Gedanken, daß sie sich auf seine Kosten bereichern und ihn, damit sie allein in seinem Hause walten könne, aus dem Wege schaffen wolle. Von dieser Idee beherrscht, beobachtete er scharf das Tun und Treiben der Frau, glaubte zu bemerken, daB seine Kinder zu ihr nehr Zuneigung zeigten, nach seiner Ansicht von ihr aufgehetzt waren. - Gegen seinen Knaben wurde er ofters aggressiv, schlug ihn und wollte die Vaterschaft desselben nicht anerkennen. Die Frau bedrohte er, stieß gelegentlich in größter Erregung die Worte aus: "Totmachen werde ich Dich nicht, aber etwas beweisen werde ich Dir." Als er ihr einmal eine Ohrfeige versetzte, glaubte er plötzlich vom Fenster her Stimmen 
zu hören, die ihn beschimpften, er sei einer solchen schönen Frau nicht wert, worauf er sie beschuldigte, daß sie "Gehilfen von allen Seiten" habe:

Am 1. II. 1911 ging T. mit seinem Sohn aus dem Hause in den an einen Fluß angrenzenden Garten, um ihm, wie er sagte, eine Fischotter zu zeigen - die Frau, die gerade dazukam, wunderte sich sehr, da in dem Wasser noch nie eine Fischotter zu sehen gewesen war - doch hatte der Knabe keine Lust mitzugehen, kehrte um und ging in die Stube zurück.

Am 5. II. ereignete sich nun folgendes: Gegen $6 \mathrm{Uhr}$ abends wurde T. im Garten stehend beobachtet, wie er lange Zeit zum Fluß hinstarrte; er kehrte dann in sein Haus zurück, ging aber bald wieder hinunter und traf bei der Haustüre mit seinem heimkehrenden Sohne zusammen. Mit den Worten: „Komm, ich werde Dir eine Fischotter zeigen," führte er den Sohn zu der Mauer, durch welche der Garten von dem Fluß abgegrenzt war. „Dort sitzt sie," meinte er und wies auf einen Stein. Nach diesen Worten hob er ihn in die Höhe, ließ ihn zunächst wieder herunter, warf ihn aber hierauf ins Wasser. Dann kehrte er in seine Stube zurück, ging nach Angaben der Nachbarn gegen seine sonstige Gewohnheit im Zimmer auf und $a b$ und rückte mit dem Stuhl; zwischen $7-8 \mathrm{Uhr}$ wurde er öfters beim Fenster stehend beobachtet, wie er auf die Straße hinausspähte; um 8 Uhr ging er, da seine Kinder nicht nach Hause kamen, mit einer Laterne auf die Straße, um sie zu suchen, kam auch vor das Haus, wohin sein Sohn, vor dem Ertrinkungstode gerettet, gebracht worden war. Durch den Menschenauflauf aufmerksam gemacht, blieb er stehen und trat auf Aufforderung des anwesenden Wachtmeisters in die Stube; als man ihm die belastenden Aussagen seines Kindes vorhielt, erklärte er: „Da weiß ich nichts davon, ich war zu Hause." Auf dem Tatorte selbst gefragt, wo die Tat geschehen sei, erwiderte er: "Ja, da weiß ich nicht."

Bei der am nächsten Tage stattfindenden gerichtlichen Einvernahme, sowie bei den weiteren Verbandlungen blieb T. hartnäckig auf seinem Standpunkte, den wir wörtlich aus den Akten folgen lassen: „Ich habe meinen Sohn Fritz nicht in den Fluß geworfen, ich habe meinen Sohn gerne gehabt und es wird mir niemand nachweisen können, daß ich ihn nicht gern gehabt hätte oder daß ich ihn mißhandelte - soviel ich weiß, ist mein Sohn ein ganz braves Kind, wahrheitsliebend und er hat nie gelogen. Es ist richtig, $\mathrm{da} B$ ich meinem Jungen vorige Woche eine Fischotter zeigen wollte, ich habe aber auch damals nicht die Absicht gehabt, den Jungen ins Wasser zu werfen. Wie mein Junge in den Fluß geraten ist und wer ihn herausgezogen hat, weiß ich nicht."

Am 21. III. 1911 wurde T. zur Klinik gebracht; hier verhielt er sich von Anfang an vollkommen ruhig, geordnet, war verschlossen, suchte keinen Verkehr. An seiner Angabe, von der Tat nichts zu wissen, hielt er unbedingt und dauemd fest. Der Mordversuch werde ihm nur ,aufgepelzt", sagte er, seine Kinder seien doch sein Stolz, die Frau, die wahrscheinlich den Buben selbst hineingeworfen hat, habe nun erreicht, was sie wollte. Er drängte beständig auf seine Entlassung, wollte sich dem Gericht stellen, damit er als unschuldig freigesprochen werde.

Patient war kein Trinker, litt an keinen Anfällen, zeigte keinerlei Intelligenzdefekt - es handelte sich hier um einen wohl als Paranoia zu qualifizierenden Fall.

II. Sch. A., Arbeiterfrau, 57 Jahre alt, verheiratet, verübte im Dezember 1912 ein furchtbares Attentat gegen die Hausbesorgerin; bei Anbruch der Dämmerung war diese im Begriffe, die Lampen im Stiegenhause anzuzünden, als plötzlich im ganzen Hause ein gräßliches Wehklagen erscholl. Die hinausstürzenden Hausbewohner sahen die Frau mit brennenden Kleidern hinablaufen und am ganzen Körper mit Brandwunden bedeckt sich im Hofe herumwälzen; später etwas beruhigt. 
sagte sie, da $B$ ihr auf dem Gang die Sch. plötzlich entgegengetreten sei, sie mit einer Flüssigkeit - dem Geruche nach zu schließen, mit Spiritus - begossen, ihre Kleider mit einer brennenden Kerze in Brand gesteckt habe und daraufh in in ihre Wohnung zurückgeeilt sei. Nach dieser Aussage wurde die Sch. sofort für verhaftet erklärt und an Ort und Stelle mit der tödlich Verletzten konfrontiert - ihr Benehmen, insbesondere ihr Leugnen angesichts ihres Opfers war so auffällig, da/3 für den Arzt kein Zweifel bestand, daß die Sch. geisteskrank war, weshalb er die Utberführung in die Irrenanstalt empfahl. Daß die Utberfallene die Wahrheit ausgesagt hatte, konnte bei Durchsuchung der Wohnung der Sch. konstatiert werden; wo tatsächlich der bezeichnete Topf gefunden wurde, der nach Spiritus roch.

Aus den Angaben des Mannes der Sch. ging hervor, da B seine Frau seit 2-3 Jahren an Verfolgungsideen litt, indem sie vermutete, daß die Leute auf der Gasse sie verspotteten, über ihr Aussehen Bemerkungen machten, sie sehe wie eine Leiche aus; im Geschäft, auf der Straße, im Hause bestand - so glaubte sie ein Verein von ihr unbekannten Leuten, die ihr alles mögliche Unangenehme zufügten. In der letzten Zeit beschuldigte sie ihren Mann, auch er sei im Komplott und gebe ihr Pulver ins Essen. In ihrer und des Mannes Abwesenheit sei in der Wohnung die Wäsche in Unordnung gebracht, dies oder jenes beseitigt. die Türklinken, Decken beschmutzt worden, nur um ihr Unannehmlichkeiten zu machen - doch stellte sie nie die Beschuldigten zur Rede, sondern beklagte sich nur dem Mann gegenüber tiber die ihr zugefügten Unannehmlichkeiten, ließ häufig neue Schlösser an der Wohnungstüre anbringen. Mit den Nachbarn, der Hausmeisterin verkehrte sie selten. Zur Mittagszeit an dem kritischen Tage war sie sehr gut gelaunt, fast übermütig; der Mann bat sie beim Verlassen des Hauses, nichts anzustellen, da sie früher öfters geäußert hatte, es wäre am besten, den Unannehm. lichkeiten ein Ende zu machen, das bedürfe nur eines Augenblicks. In der letzter Zeit schlief sie schlecht wegen Schmerzen im Unterleibe, litt häufig an Kopfschmerzen, konnte jedoch dabei ihre Arbeit verrichten. - Menstruation früher profus eine Woche dauernd. Seit dem 54. Lebensjahre Menopause. Keine hereditäre Belastung, kein Alkoholabusus.

Auf der Klinik war sie bei der Einbringung ganz ruhig, ließ alles ohne Widerstand mit sich geschehen - sprach spontan gar nichts, erschien etwas gehemmt, machte einen erschöpften Eindruck, gab willig Auskunft, war aber sichtlich verstimmt, zum Weinen neigend. Beim Examen nach dem Grund ihres Benehmens in der letzten Zeit gefragt, brach sie in Tränen aus, erklärte, sie wisse, daß sie in der letzten Zeit verstimmt gewesen sei, weil die Leute schon seit 2 Jahren alles vor ihr verheimlicht hätten, alles sei gegen sie gewesen und das habe sie sich so zu Herzen genommen. Sie habe auch deshalb ihren Mann gebeten, die Wobnung zu wechseln, weil die Leute ihr nur übelwollten; in der jetzigen Wohnung wohne sie schon 20 Jahre, habe mit niemandem einen Streit oder einen Anstand gehabt. Gesprochen wurde über sie so, daß sie es niemandem nachweisen konnte. "Wenn ich im Zimmer war, so sprachen sie draußen auf dem Gange; weil ich so ein Augenleiden und solche Schmerzen im Kopf habe, sagten sie, daB ich ins Irrenhaus gehöre, alles Mögliche sagten sie über mich, sagten auch, daß sie mir das Ärgste antun würden, damit ich mich aufhänge; alles Mögliche hatten sie gegen mich vor, (laut jammernd) Gott, was ich leiden muß, ich weiß nicht warum." Die Antworten erfolgen immer in kurzen Sätzen, etwas widerstrebend unter Tränen.

Am ärgsten trieb es ihrer Aussage nach eine Nachbarpartei, die seit 2 Jahren dort wohnt, ebenso die Parteien über ihr und unten im Parterre; sie klopften in der Nacht, damit sie nicht schlafen könnte, hielten es vermutlich mit ihrem Mann, hetzten ihn gegen sie auf, „er hat mehr zu ihnen gehalten als zu mir, er hat mir in nichts nachgeben wollen". Auf der Gasse, in den Geschäften bemerkte sie alle 
möglichen Witze, alles gegen sie; wenn sie die Türklinke ergriff, bekam sie Krämpfe in die Hand. „Die Leute behaupten, das stinke nach Leichen, das bleibe immer an meinen Händen."

Über ihre Stimmung am Tage der Tat befragt, gab sie $\mathrm{zu}$, heiter gewesen $\mathrm{zu}$ sein, sie hätte aber wenig Grund zur Heiterkeit gehabt, verstehe nicht, wie sie habe lachen können. Auf ihre in der Zeitung ihr vorgezeigte Tat verwiesen, behauptet sie, von nichts zu wissen; nach ihrer Angabe verließ sie die Wohnung, um in einem Topfe Wasser zu holen, ging dann in die Küche und zündete, da es noch finster war, die Lampe an. „Draußen börte ich wohl Lärm, doch ist das nichts Ungewöhnliches bei uns, ich machte Feuer im Herde. Vom Fenster sab ich eine Menge Leute vor dem Hause stehen, ging aber nicht hinaus. Nach einer Weile kam der Polizeikommissär, der sagte, daB mich die Hausmeisterin beschuldige und ich fragte, wessen sie mich beschuldige, ich hatte doch nur Wasser geholt. Sie führten mich zu ihr und da sah ich, daB sie schlimm daran sei." Von ihr der Tat beschuldigt, habe sie ihr gesagt: „Bitte Sie, Frau Hausmeisterin, ich habe Ihnen doch das nicht gemacht, ich habe das doch nicht tun können."

2 Tage später wurde der Sch. der Tod der Hausmeisterin mitgeteilt, was sie ruhig zur Kenntnis nahm. "Ich habe ihr nichts gemacht, ich habe gar keine Ursaehe gehabt, das zu tun (mit gefalteten Händen und salbungsvollem Tone), wir haben immer friedlich miteinander gelebt, wir haben nie einen Streit gehabt, es kann niemand, auch mein Mann nicht sagen, daß ich das Geringste gegen sie gehabt habe - noch am Nachmittag sprachen wir zusammen beim Wasserholen. (Sagt noch einige Male leise vor sich hin): „Ich babe es nicht gemacht, ich habe es nicht gemacht."

Auf diesem Standpunkt beharrte sie hartnäckig während der ganzen Dauer ihres Aufenthaltes in der Klinik.

Patientin war keine Trinkerin, hatte keine Anfälle, zeigte keinerlei Intelligenzdefekt, benahm sich vollkommen korrekt, geordnet; auch hier handelte es sich um einen paranoischen Symptomenkomplex.

III. L. M., 29jähriger Mann, keine schwerere Heredität, entwickelte sich als Kind normal, lernte mittelmäßig, war „sanfterer" Natur, litt nach dem 14. Lebensjahr an Ohrausfluß, mit darauffolgender Schwerhörigkeit. Nach der Schulzeit begann er beim Vater das Wagnerhandwerk, vervollkommnete sich darin bei einem anderen Meister und trat danach wieder bei seinem Vater als Arbeiter ein. Doch war dieșer mit der Arbeit seines Sohnes nie zufrieden, jagte ihn sogar einmal aus dem Hause. Zu Beginn des Krieges, im Jahre 1914 rückte er an die Front ein, erkrankte in den Karpathen im Anschlu $\beta$ an eine Verkühlung neuerlich an Ohren. fluß, wurde ,kopfkrank", daraufhin im Wege der Spitäler superarbitriert und schließlich als Arbeiter im Hinterland verwendet. Infolge Ausbruches einer Geisteskrankheit erfolgte im August 1916 seine Internierung im Prager Garnisonspitale; er äußerte damals Wahnideen, daß er jede Nacht elektrisiert werde und während des Schlafes eine starke Hitze verspüre, bot daneben ein stumpfes, apathisches Verhalten und wurde mit der Diagnose Dementia paranoides in eine Irrenanstalt transferiert. Noch in demselben Jahre nahm ihn der Vater von dort nach Hause, wo er durch sein menschenscheues, nachdenkliches, wortkarges Wesen auffiel, häufig über Kopfschmerzen klagte. Ab und zu half er bei der Arbeit, konstruierte selbst eine Geige, spielte darauf, obwohl er nie geigen gelernt hatte. In den letzten $11 / 2$ Jahren mied er jegliche Arbeit und gegen seine frühere Gewohnheit auch die Kirche, ging meist spazieren oder las Bücher.

Seine Schwestern liebte er, dem Vater jedoch brachte er nicht die rechte Liebe entgegen, da er von ihm oft mißhandelt und beschimpft wurde. Im Mai 1920 
nahmen die Streitigkeiten zwischen Vater und Sohn zu, da der Vater ihn, weil er nichts arbeiten wollte, zum Verlassen des Hauses aufforderte und sich gelegentlich zu einer seiner Töchter äußerte, daß der Sohn unter allen Umständen am 29. V. von Haus weg sein müsse; warum es aber an diesem Tage nicht dazu kam, ist nicht bekannt. Am Vormittag des 1. VI. nun gerieten die beiden gegen $7 \mathrm{Ubr}$ im Wohnzimmer in Anwesenheit der ältesten Tochter Christine wiederum in einen heftigen Streit, indem der Vater verlangte, daß der Sohn die Kühe weiden oder sonst etwas arbeiten solle, der Sohn hingegen sich widersetzte und erklärte, da $\beta$ er ,dienen" gehen würde, wenn ihn die Krankheit nicht daran hinderte. Dabei waren beide sehr aufgeregt und im Moment, als die Tochter Christine auf einen Augenblick das Zimmer verlieB, schlug der Sohn seinen Vater mit dem auf dem Boden liegenden Stiefelknecht in die rechte Schläfe, ging daraufhin auf den Hof hinaus, wo er mit der Schwester zusammentraf; er zitterte am ganzen Körper, war sehr bleich und erwiderte auf eine Frage der Schwester: „Ich hab' den Vater erschlagen!" Danach begab er sich auf den Dachboden und blieb trotz des Zusammenlaufens der Leute bis zum Nachmittag oben, stieg dann herunter und fragte die Schwester, ob der Vater aus der Sache „herauskommen" werde. Als er hörte, daß dies nicht der Fall sein würde, erklärte er, die Tat ungern begangen zu haben, der Vater hätte ihn nicht so aufregen sollen. Die darauffolgende Nacht brachte er wiederum auf dem Dacbboden zu, erkundigte sich am Morgen (2. VI.), wie es dem Vater gehe, ging auf die Mitteilung hin, dab der Vater gestorben sei. ins Zimmer zu ihm, erblickte die Leiche und brach in heftiges Weinen aus. Als er an demselben Tage verhaftet und von den Gendarmen über die Tat befragt wurde; sprach er so unsinnige Sachen, daß er uber Weisung des anwesenden Arztes als. geisteskrank nach Prag überführt wurde.

Während seines Aufenthaltes in der Klinik bot er ein vorwiegend stumpfes, apathisches Verhalten, micd den Verkehr mit den anderen Kranken, gab beim Examen an, daß der Krieg seine Nerven vollständig zugnunde gerichtet habe; er bekomme, so oft er arbeiten wolle, Hitze im Kopf, werde von jemandem mit elektrischen Strömen verfolgt, spüre sie zeitweise in der Hand, in den Füßen, im ganzen Körper. Über die Tat befragt, erklärte er mit Entschiedenheit, er wisse nichts davon, er habe seinen Vater nie geschlagen, auch nie mit ihm gezankt. Wohl aber habe ihn der Vater immer schlecht behandelt, habe ihn damals aufgefordert, die Kühe zur Weide zu führen. „Ich habe mich nicht gestritten, war nicht aufgeregt, habe zu ihm nur paar Worte gesagt, das war kein Streit. Ich habe ihm nur gesagt, er soll nicht so sein, daß ich nicht arbeiten könne. Ich habe ihn nicht mit dem Stiefelknecht geschlagen. Es ist nicht wahr, daß ich der Schwester Marie sagte, ich habe den Vater erschlagen. Marie kann das doch nicht angeben, sie war nicht zu Hause. Ich weiß nichts anderes zu sagen, als daß die älteste Schwester, als ich dann ins Zimmer kam, dort war, der Vater am Bette lag und von meinem Cousin entkleidet wurde. Ich war an diesem Tage nicht ohne Bewußtsein, so etwas war bei mir nicht der Fall. Daß ich nicht gewußt hätte, was ich tue, das gebe ich nicht zu. In der Wirtschaft hat der Vater eine derartige Nachlässigkeit an den Tag gelegt, daß er "nicht ganz gescheit" gewesen zu sein scheint. Seine beiden Frauen hat er ärger wie ein Trunkenbold, obgleich er nicht getrunken hat, geprügelt und geschlagen, so daß die Mutter einmal auf eine Woche vor ihm fliehen mußte." Auch seien Streitigkeiten und Zusammenstöße mit anderen Leuten oft und oft vorgekommen.

Es handelt sich in diesem Falle um eine Dementia paranoides und damit mag es zusammenhängen, daß im Gegensatze zu den vorher mitgeteilten Fällen dieser Kranke zunächst seine Tat gesteht und erst später ableugnet. 
Die forensische Bedeutung der Amnesie und ihre Erklärung usw.

IV. H. A., 52 Jahre alt, war als Kind gesund, lernte in der Schule mittelmäßig, war über 20 Jahre bei der Bahn bedienstet und wurde angeblich wegen eines im Jahre 1911 erlittenen Unfalles.- nach Angabe der Frau soll er einen Kameraden mit dem Revolver bedroht haben und dies der Grund seiner Entlassung gewesen sein - pensioniert. Seitdem zu Hause auffällig, mied er seine Angehörigen, wollte von ihnen nichts wissen, bedrohte sie ständig mit Messer oder Hacke, äußerte, ,er werde allen den Schädel zerschlagen, sie ins Jenseits schaffen, alle Leute im Dorfe seien gegen ihn verbunden, um ihn zu verniehten."

Dieser Zustand steigerte sich allmählich, er wurde auf Veranlassung seiner Frau im März 1919 in der Irrenanstalt interniert, wo er alle diese Drohungen in Abrede stellte und angab, daß die Hauptschuld an den häuslichen Streitigkeiten seine Frau und deren Stiefschwester tragen, die ihn beschimpft hätten und aus dem Hause werfen wollten. Die Frau sei schlecht $\mathrm{zu}$ ihm gewesen, sei von einem Lehrer gegen ihn ,aufgehetzt" und habe ihn immer wieder ,erniedrigt". Mit den anderen Leuten sei er gut ausgekommen, doch habe er auch an ihnen bemerkt, daB sie gegen ihn eingestellt oder aufgewiegelt seien, weil sie seine Begrüßung nicht erwiderten.

In Mai 1919 wurde er auf Ansuchen der Frau in gebessertem Zustande aus der Anstalt entlassen, unter Kuratel gestellt und seine Frau zum Kurator bestimmt. Infolge dieser Bevormundung fühlte sich $\mathrm{H}$. in seiner Freiheit eingeschränkt und versuchte durch Vermittlung von Adrokaten die Aufhebung seiner Eritmündigung durchzusetzen, was ihm jedoch nicht gelang. Darüber war er sehr aufgebracht, die Streitigkeitèn im Hause nahmen zu und häuften sich. Er schlug wiederholt die Tochter und die Frau, bedrohte sie und die Gerichtsbeamten, die die Kuratelaufhebung abgelehnt hatten, mit Ermorden, äußerte gelegentlich: „Wenn ich diese Kerle beisammen hätte, möchte ich sie umbringen."

Am 16. X. 1920 sprach er davon, daß er jemanden umbringen müsse, daß auch die Frau seiner Hand nicht entgehen werde, äußerte: „Du entgehst mir nicht, dessen kannst du sicher sein "

Am 17. X. war er bis 6 Uhr abends zu Hause, suchte dann ein Gasthaus auf, trank an diesem Abend 2 halbe Liter Bier, während er sonst nur $1 / 2$ Liter zu trinken pflegte und ging etwa um $7 \mathrm{Uhr}$ von dort fort. Dem Wirt gegenüber erklärte er, $\mathrm{da} B$ er auf seine Frau, die um Lebensmittel gegangen sei, warten müsse. (In Wirklichkeit war die Frau zu Hause gewesen und hatte an diesem Abend die Wohnung nicht verlassen.) Auf dem Wege begegnete er einer ihm unbekannten Frau, die einen Knaben auf dem Arm trug, schoß von weitem auf sie und verletzte sie leicht am rechten Ellbogen. Dann kam er näher, packte den Knaben von rückwärts am Kragen, fragte, ob er sich das Hündehen, das gerade auf der Straße lief, anbinden wolle oder nicht und ging auf der Straße weiter. Gegen $3 / 48 \mathrm{kam}$ er nach Hause, legte sich nieder, rauchte noch ein wenig und schlief dann ein. Am nächsten Tage verabschiedete er sich von seiner Frau, erklärte, er fahre in der Angelegenheit der Kuratelaufhebung fort und, falls er zu seinem Rechte kommen sollte, kehre er wieder zurück. Am nächsten Tag jedoch kam er wieder nach Hause, worde kurz darauf in die Gemeindekanzlei gerufen, trotz seiner Bitten und Einwände, daß er sich doch keiner Schuld bewußt sei, verhaftet und am 21. X. in der Klinik interniert.

Während seines hiesigen Aufenthaltes zeigte $\mathrm{H}$. ein ruhiges, geordnetes, etwas stumpfes Verhalten, gab auf Befragen genau Auskünfte über seine häuslichen Verhältnisse, erklärte entschieden, mit seiner Frau keine Streitigkeiten gehabt, sich mit jedermann vertragen zu haben; die Frau sei es gewesen, die ihn schon den 3. Tag nach der Hochzeit geschlagen und beleidigt habe, sie sei darin von einer Schwester und deren Kameradinnen womöglich noch bestärkt worden. Darum habe 
er sie gemieden, sei dann im Jahre 1919 infolge verschiedener Ränke seiner Frau unter Kuratel gestellt und seit dieser Zeit mit neuerlicher Internierung bedroht worden. Er habe beim zustehenden Bezirksgerichte entsprechende Schritte zur Aufhebung seiner Kuratel unternommen (tatsächlich), doch seien der Gerichtsrat, und nicht bloß die eine Abteilung, mit der er zu tun hatte, sondern das ganze Gericht gegen ihn gewesen.

Den Mordversuch stellte er, so oft er verhört wurde, mit Entschiedenheit in Abrede, er habe niemanden angeschossen, habe auch nie einen Revolver bei sich gehabt, übrigens kenne er die Frau, die er angeblich verletzt habe, überhaupt nicht, sie habe noch nie ein Wort mit ihm gesprochen. Am Abend der vermeintlichen Tat habe er 2 Biere im Gasthause getrunken, doch habe es ihm diesmal nicht geschmeckt, er sei dann nach Hause gegangen, habe seine Frau in der Wohnung angetroffen und sich ins Bett niedergelegt. Nächsten Tag sei er dann vom Hause fort, habe die Absicht gehabt, in Angclegenheit der Kuratelaufhebung etwa eine Woche auszubleiben und gleichzeitig sich nach einer Arbeit umzuschauen. Da er aber keine gefunden habe, sei er nächsten Tages wieder nach Hause zurïckgekehrt und gleich am nächsten Mittag festgenommen worden. "Und ich fragte, warum ich zu Gericht gehen sollte und sie sagten mir, daß ich das wisse, doch glaubte ich, $\mathrm{da} \beta$ es sich darum handle, da $B$ man die Vormundschaft aufheben wolle. Da man mir die Hände mit Ketten zusammenbinden wollte, wehrte ich mich, doch haben sie mich trotzdem eingesperrt und mich am nächsten Tage in eine $Z_{w a n g s j a c k e}$ gesteckt. Sie sind mit mir wie mit einem Mörder umgegangen, ohne mich vorher, wie es sich gehört hätte, einem entsprechenden Verhör unterzogen zu haben."

Es handelt sich in diesem Falle um eine Paranoia bei einem etwas beschränkten Manne.

Zunächst lag der Zweifel, ob man es wirklich mit einer einfachen Amnesie zu tun hatte oder ob es sich um Simulation handelte, nahe. Die Verbrecherwelt kennt den Wert der Amnesie, die für Psychose spricht, und der Verdacht einer Simulation wird daher stets rege und bei dem paranoischen Verbrecher womöglich noch verstärkt, der durch sein mißtrauisches, verschlossenes Benehmen meistens Mißfallen erregt. Dazu kommt der Mangel sonstiger Merkfähigkeitsstörung, der gewiß nicht dazu angetan ist, die Frage der Simulation auszuschalten.

Wenn auch Dercum ${ }^{1}$ ) es in einer Diskussion hinsichtlich der Stichhaltigkeit der von vielen Verbrechern behaupteten Amnesie als Faktum hinstellt, daß die wirklich Geisteskranken niemals eine solche Angabe machen, so kann das nach Ausweis der vorliegenden Mitteilung nicht als allgemein giltig anerkannt werden und scheint besonders für die Paranoiagruppe keineswegs zuzutreffen.

Im Gegenteil, die Echtheit der die Amnesie betreffenden Angaben, die als Resultat der langen Beobachtungszeit hervorgeht, ist in keinem der Fälle zu bezweifeln. Die ehrliche Entrüstung, die die Patienten beim oftmaligen Verhör über die Zumutung, ein derartiges Verbrechen begangen zu haben, zeigten, konnte nicht gespielt sein. Der muß ein guter Schauspieler sein, der auf der Bühne niemals aus seiner Rolle fällt, die Maske,

1) Transact. Am. Neurol. Assoc. 1915, p. 33. 
Die forensische Bedeutung der Amnesie und ihre Erklärung usw. 751

unter der er spielt, nie fallen läßt, sich nie vergißt - der hält den Schein für Wirklichkeit, er spielt nicht bloß, er ist auch, was er scheint. Diese Kunst trifft im allgemeinen der Ungeübte nicht, und ein jeder wird sich wohl die Frage, ob er im stande wäre, ein tatsächlich begangenes Verbrechen bei unzähligen Verhören, stets mit der gleichen entrüsteten Miene zu leugnen, mit einem Nein beantworten, wobei noch berücksichtigt werden muß, daß der lange der Beobachtung dienende Aufenthalt in der Irrenanstalt nicht der angenehmste zu nennen ist.

Was nun die hier mitgeteilten Fälle betrifft, so liegen die Verhältnisse nicht in allen gleich und werden wir zu versuchen haben, den Mechanismus des „Vergessens" im Einzelnen klarzulegen.

In dem ersten Falle ist es das Mißtrauen gegen die eigene Frau, die den Mann absichtlich bei einem Hausbau durch Verweigerung jeglicher pekuniärer Beihilfe in Zahlungsschwierigkeiten geraten läßt, ihm seine eigenen Kinder abspenstig macht, ihn ,,angeblich" ständig kontrolliert, ein Mißtrauen, das, ins Krankhafte gesteigert, ihn schließlich Drohungen ausstoßen, handgreiflich werden läßt.

Eine Affekthandlung anzunehmen, wäre in diesem Falle nicht am Platze, da der Kranke schon den Tag vorher den Jungen ins Wasser werfen wollte, demnach vorbedacht handelte. Dadurch, daß er erst nach längerer Zeit zur Klinik kam, wird sich eine sichere Entscheidung bezüglich der Frage der Amnesie nicht fällen lassen; die Differenz zwischen der anfänglichen einfachen Ableugnung der Tat und der späteren Äußerung, daß wahrscheinlich die Frau den Jungen ins Wasser geworfen habe, lassen wohl die Möglichkeit offen, daß es sich bei diesem Kranken, bei dem ein ausgesprochen querulatorisches Moment deutlich vorliegt, um die von Wernicke so genannte negative Erinnerungsfölschung handelt, die sich aber vielleicht erst nachträglich entwickelt hat. Vit diesem Ausdrucke ,negative oder substraktive Erinnerungstäuschung". soll gesagt sein, daß der Kranke nicht bewußt absichtlich unter dem Einflusse seiner Vorstellungen die wirklichen Vorgänge, u. zw. eben diejenigen, die seinem ganzen Gedankengange widersprechen, $\mathrm{ihm}$ unangenehm sind, verdrängt, indem der unbewußte Gefühlsfaktor allmählich eine auslöschende Wirkung ausübt.

Wernicke $^{1}$ ). und Hitzig weisen in der Literatur auf derartige Vorgänge beim Querulantenwahn hin und auch beim sog. Ganserschen Symptom ist man allgemein zur Ansicht gelangt, daß ,das Nichtmehrwissen teils ein unbewußtes, teils ein halbbewußtes Nichtwissenwollen war" ( Riklin) oder wie Freud sich ausdrückt: „Die Hysterischen wissen nicht, was sie nicht wissen wollen".

1) Wernicke, Grundriß der Psychiatrie 1900. 
Dem zweiten Falle liegt ein seit mehreren Jahren sich entwickelndes Wahnsystem zugrunde; die Frau glaubt sich auf verschiedene Weise und in immer zunehmendem Maße von den Nachbarsleuten, den Leuten auf der Straße beeinflußt, benachteiligt, verhöhnt, ist dadurch sehr niedergedrückt, beklagt sich oft bitter ihrem Manne gegenüber über die ihr zugefügten Unannehmlichkeiten. Hier wird die Annahme berechtigt sein, daß es sich tatsächlich um eine Amnesie handelt, die wahrscheinlich durch die auslöschende Wirkung des großen Affektes, in dem die Tat zu rascher Ausführung kam, bedingt ist. Da auch der normale Affekt nicht selten eine, wenn auch nur minime Bewußtseinsstörung, bezw. Bewußtseinseinengung hervorruft, muß man folgern, daß ein ins Ungeheuerliche oder ein krankhaft gesteigerter Affekt, wie er auf dem prädisponierenden Boden der paranoiden Erkrankung fast immer eintritt, tempore delicti Amnesie u. zw. unmittelbar nachher zur Folge haben kann; einen besonderen Mechanismus dabei anzunehmen ist überflüssig, die hochgradige Affekterregung ruft einen Schock hervor, unter dessen Wirkung das Vergessen zustande kommt (akute Amnesie).

In unserem dritten Falle, in dem der Sohn an einem Vormittag seinen Vater mit einem Stiefelknecht erschlägt, kurz darauf die Tat seiner Schwester gegenüber ohne Zögern eingesteht, noch am Nachmittag mit sichtlicher Reue, die Tat ungern begangen zu haben, erklärt und erst am Morgen des nächsten Tages, nachdem er sich vorher nach dem Befinden seines Vaters erkundigt hat und beim Anblick der Leiche in heftiges Weinen geraten ist, für seine Handlung Amnesie zeigt, tritt die verspätete Entwicklung der Amnesie in den Vordergrund, die, wenn auch die jahrelangen, ständigen Mißhandlungen und Drohungen des Vaters ihn, den sich arbeitsunfähig fühlenden, menschenscheuen Kranken, falls er nichts arbeiten würde, aus dem Hause zu werfen, aufregten und schlieBlich zum Widerstand reizten, die Erklärung einer Affekthandlung von vornherein als unwahrscheinlich erscheinen läßt. Da wäre zunächst Freuds ,,besonderer mißgünstiger psychischer Faktor in Betracht zu ziehen, der sich dagegen sträubt, etwas zu produzieren, was Unlust entbindet oder in weiterer Folge zur Unlustentbindung führen kann", doch liegt diesmal das Verhältnis offenbar so, daß die seiner Dementia paranoides zukommende gemütliche Verstumpfung das anfängliche Geständnis und den daran anschließenden Affekt sehr bald zurücktreten lassen, was auch bei sonstigen Handlungen derartiger Kranker nicht selten zur Beobachtung kommt. Ich erwähne hier zwei Fälle der Klinik, von denen der eine das Zerschlagen einer Fensterscheibe nachher absolut nicht zugeben wollte, während ein anderer, obgleich er kurz vorher gefrühstückt hatte, mit der Motivierung, kein Frühstück bekommen zu haben, ein zweites Frühstück verlangte. 
Die forensische Bedeutung der Amnesie und ihre Erklärung usw. 753

Im vierten Fall wiederum war ein seit 'anger Zeit bestehendes Wahn. system vorhanden, in welchem der Kranke durch die anscheinend schlechte Behandlung von seiten seiner Frau immer mehr bestärkt und durch die oftmalige Bedrohung ihrerseits mit seiner Internierung in der Irrenanstalt derart in Erregung versetzt wurde, daß er menschenscheu wurde, sich von seiner Familie und Umgebung gänzlich zurückzog und Rache- und Mordgedanken nährte.

In diesem letzten etwas merkwürdigen Falle käme in der Tat am ehesten ein einfaches Ableugnen in Frage, doch gibt der Umstand, daß der Kranke gegen eine ihm ganz fremde Frau einen Attentatsversuch verübte und zu deren Kind scheinbar ganz sinnlose Worte äußerte, dahin zu denken, daß hier im Sinne Freuds eine Symbolhandlung vorlag, die später der Verdrängung anheimfiel. Für die Annahme eines pathologischen Rausches waren keine Anhaltspunkte vorhanden, ebenso konnte die Tat als eine Affekthandlung nicht in Betracht gezogen werden.

Die Erklärung des Mechanismus des „Vergessens" zeigt sich demnach in jedem der erwähnten Fälle ganz verschieden und deutet auf die Schwierigkeiten hin, mit welchen die Beurteilung der als Amnesie imponierenden Erscheinungen bei paranoiden Erkrankungen verknüpft zu sein pflegt. Die vorliegende Arbeit hat den $Z_{w e c k}$, einesteils auf die hohe forensische Bedeutung derartiger Mechanismen hinzuweisen, anderenteils weitere Beobachtungen in dieser Richtung bei paranoiden Erkrankungen anzuregen. 